\title{
A Conceptual Approach to Urban Wellbeing from a Human Evolution Perspective
}

\author{
Gustav Milne \\ UCL Institute of Archaeology, 31-4 Gordon Square London WC1 H OPY UK: g.milne@ucl.ac.uk \\ Museum of London Archaeology, 46 Eagle Wharf Road London N1 7ED UK: gmilne@mola.org.uk
}

\begin{abstract}
To cope with a projected global population increase from 7.2 bn to 9.6 bn by 2050, many more cities must be built. Although there are great benefits to modern urban living, there also great costs, such as the seemingly unstoppable rise in Type 2 diabetes, obesity, coronary issues and various cancers. The new towns should be designed to contain or constrain the epidemic of those 'Western Lifestyle Diseases' that currently plagues today's cities. But how might this be achieved?
\end{abstract}

It is suggested here that a greater understanding of human evolution combined with the potency of the 'Palaeolithic genome' holds the key to our future urban wellbeing. Consequently, a new paradigm is suggested that underpins positive forward thinking on townplanning and city lifestyles to create healthier urban environments. This builds directly on the 'Evolutionary Determinants of Health' programme initiated at University College London (UCL). A four-stage model is proposed that integrates and develops both evolutionary-concordant personal and institutional health behaviours with appropriately reconfigured townplanning and building regulations. When integrated, these strands could deliver a healthier urban culture within greened, active townscapes by proactively constraining or eliminating some of the key underlying causes of the so-called 'Western Lifestyle Diseases'.

Keywords: conceptual model; Evolutionary Determinants of Health; greened city; human evolution; Palaeolithic genome; urban greenspace; urban wellbeing; Western Lifestyle Diseases

\section{INTRODUCTION}

For the first time in human history, more people now live in towns than in rural communities. The continuing march of modernity has urbanisation at its core, and to cope with a projected global population increase from $7.2 \mathrm{bn}$ to $9.6 \mathrm{bn}$ by 2050 , many more cities must be (and are being) built. Although there are great benefits to modern urban living, there also great costs. These include a raft of health issues such as the seemingly unstoppable rise in Type 2 diabetes, obesity, coronary issues and various cancers. The new towns of the future should be designed to contain or constrain the epidemic of those 'Western Lifestyle Diseases' that currently plagues today's cities.

But how might this be achieved? There has been considerable research on aspects of urban health, as a recent review in this journal has shown ${ }^{1}$. The studies discussed considered the relationships between individuals and society as well as between the natural and the built environment, and the complex web of interactions that resulted. To these were added cultural, age and wage differentials ${ }^{2}$ to further the complexity. That issues such as building design ${ }^{3}$ access to green space ${ }^{4}$ air quality ${ }^{5}$ and excessive car use ${ }^{6}$ also have a profound impact on

\footnotetext{
${ }^{1}$ von Szombathely et al 2017 von Szombathely, M., Albrecht, M., Antanaskovic, D., Augustin J., Augustin, M., Bechtel, B., Bürk, T., Fischereit, J., Grawe, D., Hoffmann,P., Kaveckis. G., Krefis. A., Oßenbrügge. J., Scheffran. J. and Heinke Schlünzen. K., 2017 'A Conceptual Modeling Approach to Health-Related Urban Well-Being' Urban Sci. 2017, 1, 17; doi:10.3390/urbansci www.mdpi.com/journal/urbansci

2 eg Liddle 2017) Liddle, B., 2017 'Urbanization and Inequality/Poverty' Urban Sci., 1(4), 35; doi:10.3390/urbansci1040035 - 27 Nov 2017

3 eg Smith et al 2013), Smith, L, Ucci, M, Marmot, A, Spinney, R, Laskowski, M, Sawyer, A, Konstantou, M, Hamer, M, Ambler, G, Wardle, J and Fisher, A., 2013 'Active Buildings: modelling physical activity and movement in office buildingsan observational study protocol', BMJ Open 2013;3:e004103. Doi:10.1136/bmjopen-2013-004103

${ }^{4}$ Mitchell, R., and Popham, F., 2008 'Effect of exposure to natural environment on health inequalities: an observational population study', The Lancet 372(9650), 1655-1660
} 
urban wellbeing is increasingly evident. Poor health is often easy to identify, although effective remedies for a particular condition will depend on the complex interactions previously discussed.

But to take a step back, the Evolutionary Determinants of Health programme ${ }^{7}$ initiated at University College London considers, not so much how, but why so many elements of urbanisation have a negative effect on our health. It then argues for proactive, upstream preventive strategies that address this challenge ${ }^{8}$. Some of the fundamental issues considered in this project relate to our basic biology, which cannot be changed, and urban morphology, which, being an artificial creation, can be changed.

\section{NORMAL LIVES}

Some $7 \mathrm{~m}$ years ago, our human lineage began diverging from that of the chimpanzee, with whom we still share $98 \%$ of our genome and thus a common Miocene ancestor ${ }^{9}$. For the majority of the subsequent period, our basic biology gradually evolved, as our various direct ancestors lived off the land, developing into triballybased 'hunter-gatherer-style' communities. That protracted period in our human evolution is stamped deep into our DNA as our genetic 'normal'.

Such active 'hunter-gatherer-style' cultures, robustly refined by the rigours of natural selection, are well adapted to our bi-pedal physiology, while our dentition, metabolism and digestive system are likewise well suited to a diet of fresh fruit, vegetables, roots, nuts, berries, meat and fish ${ }^{10}$. There have been many detailed studies of the benefits afforded by 'ancestral' diets, beginning with the pioneering approach taken by Weston Price $^{11}$, the research by Melvin Konner and S. Boyd Eaton ${ }^{12}$ and the remarkable work by Staffan Lindeberg ${ }^{13}$, for example.

The human race is genetically adapted for a life of routine light to moderate activity essential for survival (walking, lifting, carrying, bending, climbing), rather than for long sedentary periods ${ }^{14}$. The actual tasks accomplished in a "normal" hunter-gatherer's day could vary, depending on the level of hunger, seasonality, weather or terrain. Nevertheless, it seems that the typical daily distance covered by human locomotion would be in the range of three to ten miles. The necessary daily activities would require an average energy expenditure of between 3,000 and $5,000 \mathrm{kj}$, up to five times greater than many modern sedentary adults ${ }^{15}$. For today's city dwellers, regular walking or cycling at least part of the way to and from work/school each day would be a modest evolutionary-concordant compromise, given an encouraging townscape to move within ${ }^{16}$.

In addition, the lives of hunter-gatherers were lived outdoors within a natural environment. Crucially, we are

\footnotetext{
${ }^{5}$ DEFRA 2015 Improving Air Quality in the UK: Tackling Nitrogen Dioxide in Our Towns and Cities. UK Overview Document. London: Department for Environment, Food and Rural Affairs

6 Hynes, M., 2017 'At a Crossroads: Investigating Automobility and Its Implications for Local Urban Transport Policy Design' Urban Sci. 2017, 1(2), 14; doi:10.3390/urbansci1020014 Published: 5 May 2017

7 Milne, G., 2015 'The Evolutionary Determinants of Health Programme: urban living in the $21^{\text {st }}$ century from a human evolutionary perspective' Archaeology International 18, 84-96 DOI: http://dx.doi.org/10.5334/ai.1809)

${ }^{8}$ for further details, case studies and references, see Milne, G., 2017 Uncivilised Genes: human evolution and the urban paradox, Crown House Publications, Carmarthen

9 Stringer, C. and Andrews, P., 2011 The Complete Word of Human Evolution. London: Thames \& Hudson

${ }^{10}$ Kelly, R., 2013 The Lifeways of Hunter-Gatherers: The Foraging Spectrum. Cambridge: Cambridge University Press; see also Milne, G., 2017 Uncivilised Genes: human evolution and the urban paradox, 225-228

${ }^{11}$ Price, W., 1939 Nutrition and Physical Degeneration: A Comparison of the Primitive and Modern Diet and Their Effects. Redlands, CA

12 Konner, M. and Eaton, S. B., 2010 'Palaeolithic nutrition: 25 years later', Nutrition in Clinical Practice 25, 594-602

${ }^{13}$ Lindeberg, S., 2010 Food and Western Disease: Health and Nutrition from an Evolutionary Perspective

${ }^{14}$ O’Keefe, J., Vogel, R., Lavie, C. and Cordain, L., 2011 'Exercise like a hunter-gatherer: a prescription for organic physical fitness', Progress in Cardiovascular Diseases 53, 471-479

${ }^{15}$ Cordain, L, Gotshall, R and Eaton, SB., 1998 Physical Activity, energy expenditure and fitness: an evolutionary perspective. Int J Sports Med 19: 328-335.

${ }^{16}$ Eg Marshall, S., Milne, G., Rook, G. \& Tinnerman, R., 2015 'Walking: a step-change towards healthy cities', Town and Country Planning Journal, 125-129; Wang, L and Wen, C., 2017 'The Relationship between the Neighborhood Built Environment and Active Transportation among Adults: A Systematic Literature Review' Urban Sci. 2017, 1(3), 29; doi:10.3390/urbansci1030029 Published: 27 Aug 2017
} 
not born with a fully-functioning immune system, since this co-evolved with us over the long period of human evolution as Professor Graham Rook's ground breaking studies have shown ${ }^{17}$. It is now clear that it is from direct contact with plants, animals and other humans that we obtain the macro-organisms, microorganisms and microbiota that live and thrive on our skin and in the gut, managing our immune system within our own personal ecosystem. We are not born with these microbiota: initially, we derive them from our mother's birth canal but not, alas, from a Caesarean section ${ }^{18}$. Subsequently we absorb these organisms from the external environment, from the soil, from plants, trees and animals, from the air. Without them, we are increasingly susceptibility to allergies, autoimmunity and inflammatory bowel disease. Consequently, reduced contact with nature is bad for our physical health: we still need the microorganisms that only the natural environment can provide. Living in sterile urban areas, however, decreases our exposure to nature (and thus a less effective immune system) while urban life increases exposure to crowd infections.

\section{DISEASES OF URBANISATION}

Culturally, the human race has seen dramatic and rapid transformations. Genetically, however, our evolution has been much more gradual: anatomically we remain much as we were before extensive agriculture and urbanisation were gradually developed in the Neolithic period, some 5,000 to 10,000 years ago. Although there are major benefits in city living, there are also major costs, such as the seemingly unstoppable rise in obesity, coronary-related problems, Type 2 diabetes, Alzheimer's and various types of cancer. According to the World Health Organisation, these are all listed in the ten most common causes of death in modern, urbanised societies. Do these deaths represent that mismatch between human biology and urban culture, or are they just an inevitable result of the ageing process?

There is, in fact, compelling research that challenges the inevitability of death by 'Western Life-style Diseases' and raises the possibility that living in such artificial built environments may well be exerting an unanticipated but negative impact on modern urban wellbeing. A major long-term study by Dr Staffan Lindeberg, for example, has shown that ALL those fatal 'Western' conditions are rare or non-existent in un-urbanised communities still maintaining an 'ancestral' life-style. His detailed research, included results from a long-term study of a large community in Kitava, Papua New Guinea, where some of those people lived well into old age ${ }^{19}$.

Archaeological research supports this assumption, following the many studies of ancient cemeteries and related research graphically showing how the transformation from ancestral practices to farming and urbanisation damaged our collective wellbeing. The domestication of plants and animals during the Neolithic period, for example, heralded major changes in the human diet and activity regimes, and thus in our wellbeing. The introduction of intensive farming saw a noted increase in dental caries, dental defects, tooth loss, trauma, metabolic and joint disease. It is also associated with the first evidence of tumours, anaemia, diffuse idiopathic skeletal hyperostosis (DISH, a proxy for obesity) and osteoporosis, as well as infections such as osteitis and periostitis of sinuses, ribs and skull. Several centuries later, the Romans not only introduced the civilising concept of urbanisation to these islands, but also scurvy, osteomalacia (rickets), Reiter's syndrome, gout, ankylosing spondylitis, rheumatoid arthritis, psoriatic arthritis, septic arthritis, tuberculosis, poliomyelitis and

\footnotetext{
${ }^{17}$ Rook, G., 2012 'Hygiene hypothesis and autoimmune diseases', Clinical Reviews in Allergy \& Immunology 42(4), 1-15; Rook, G., 2013 'Regulation of the immune system by biodiversity from the natural environment: an ecosystem service essential to health', Proceedings of the National Academy of Sciences of the USA 110(46), 18360-18367 DOI: 10.1073/pnas.1313731110; Rook, G., Raison, C. and Lowry, C., 2013 'Childhood microbial experience, immunoregulation, inflammation and adult susceptibility to psychosocial stressors and depression in rich and poor countries', Evolution, Medicine, and Public Health 1, 14-17. DOI:10.1093/emph/eos005; Rook, G., Raison, C. and Lowry, C., 2014 'Microbial "old friends", immunoregulation and socio-economic status', Clinical and Experimental Immunology 177, 13-23. DOI: 101111/cei.12269

${ }^{18}$ Yuan, C., Gaskins, A., Blaine, A., Zhang, C., Gillman, M., Missmer, S., Field, A. and Chavarro, J., 2016 'Association between cesarean birth and risk of obesity in offspring in childhood, adolescence, and early adulthood', JAMA Pediatrics. Published online 6 September. DOI: 10.1001/jamapediatrics.2016.2385
}

19 Lindeberg, S., 2010 Food and Western Disease: Health and Nutrition from an Evolutionary Perspective 
leprosy. None of these diseases or conditions were seen in the largely un-urbanised populations that lived here before the Roman invasion of AD 43. Arguably, urbanisation was -and often remained- a mixed blessing ${ }^{20}$. Certainly un-urbanised or pre-urbanised populations also suffered from problems, such as malaria, childbirth complications, predators, seasonal food or water shortages and serious accidents associated with robust active lives. But what they did NOT suffer from were many of the aliments straining our health services today: these were arguably introduced by urbanisation through its culture and its townscapes.

\section{Defining moment}

However it is measured, there is now a general consensus that the maintenance and promotion of physical and psychological wellbeing for the entire population is a precondition of a more prosperous and cohesive society. For the purposes of this report, the basic question must therefore be asked and answered: how do we define 'good health' and 'wellbeing', as well as their contrary states 'ill health' and 'ill-being'? Peace has been described as the absence of war, based on the assumption that neither of those two elements represented normality: life was ever in flux, moving inexorably from one to the other. But good health and ill health need not be seen in the same light, each being defined solely as the absence of the other. A more positive, proactive line is suggested here, that good health be considered as the expected norm and ill health the abnormal state. The constitution of the World Health Organization, as adopted in New York in June 1946, defined health not just as the absence of disease or infirmity, but as a state of complete physical, mental and social wellbeing. In 2008 , it was suggested that wellbeing should also include how people feel and function ${ }^{21}$. Those definitions are carried forward and expanded here, accepting that our basic physiology, metabolism and mindset are all determined by our long human evolution. This shows us what we were (and still are) genetically best adapted for, and, just as crucially, what we were not best adapted for. We are well adapted, for example, to eat fresh food and take daily exercise. Our overburdened National Health Service is all too well aware of the complications that arise from an urban population that ignores these fundamental evolutionary determinants of health, as the incidence of obesity, diabetes, cardiovascular problems and several forms of cancer all too painfully prove. As Professor Marmot states, "Although our material and social environments have changed beyond recognition over the last 10,000 years ... our underlying biology is essentially the same as it was in ancient Babylon" ${ }^{22}$. Archaeologists heartily agree with his sentiment, but would respectfully add many millennia to his chronology.

Here, wellbeing and good health (physical and mental) are defined as the product of following evolutionaryconcordant behaviours. III health is partially the converse, a lifestyle that deviates from that broad path and is impacted by the associated consequences, or one that is affected by trauma or by infectious or contagious diseases, a common occurrence in conurbations.

Normality in health terms is working with our Palaeolithic genome; poor health is therefore abnormal, too often the product of a lifestyle that is not evolutionary-concordant.

Good health is the consequence of adopting normal evolutionary-concordant behaviour - for example, the proxy nutritional regimes, societal systems and lifestyle-embedded activities that all correspond to and are required by that concept.

Urban wellbeing is a state of mind and body obtained by those who have adopted evolutionary-concordant behaviours within an urban environment modified on evolutionary-concordant lines ${ }^{23}$.

\footnotetext{
${ }^{20}$ see eg Roberts, C. and Cox. M., 2003 Health and Disease in Britain, from Prehistory to the Present Day. Stroud: Suton Publishing; Hassett, B., 2017 Built on Bones:15,000 years of life and death. London. See also see Milne, G., 2017 Uncivilised Genes: human evolution and the urban paradox, 229-232

${ }^{21}$ Abdallah, A., Steuer, N., Marks, N. and Page, N., 2008 Well-Being Evaluation Tools: A Research and Development Project ,for the Big Lottery Fund Final Report. London: New Economics Foundation, 7-8

22 Brunner, E., and Marmot, M., 2006 Social Determinants of Health. Oxford: Oxford University Press, 13

${ }^{23}$ Milne, G., 2017 Uncivilised Genes: human evolution and the urban paradox, Crown House Publications, Carmarthen, 20
} 


\section{ADDRESSING THE CHALLENGE}

The megacities of the future should be designed to contain or constrain the epidemic of 'Western Lifestyle Diseases' that currently beset today's towns. These and a myriad of other issues have been considered at length by bodies such as the Healthy Cities Movement ${ }^{24}$ and the World Health Organisation ${ }^{25}$. As a contribution to this complex and far-reaching debate, it is suggested here that the urban lifestyles and townscapes of tomorrow should simulate, or at least approximate, the diet, activity regimes and environments that our hunter-gatherer biology would respond to as 'normal'. Such a situation would create the basic conditions for normal good health and encourage urban wellbeing. A series of 'Protocols' are being developed to open the debate, providing an 'upstream' approach to containing the increase in 'Western lifestyle diseases' through cultural and behavioural change, from a human evolutionary perspective. The suggested mechanisms operate on four intergrated levels, initially with personal and institutional protocols, supported by town planning and building design protocols and then, at the next level, the political protocol

\section{Eden Protocol}

Lifestyle choices are moulded by a complex of cultural, economic and societal influences, while the associated research incorporated studies concerning physiological, metabolic and psychological factors relating to nutrition, activity regimes and social interactions. The Alameda County Study undertaken by the epidemiologist Sir Richard Doll, considered a cluster of lifestyle factors which were related over time to a higher or lower risk of death: these were termed 'positive' and negative' health behaviours ${ }^{26}$. In a similar vein, the evolutionary determinants of health programme has identified 'normal' evolutionary-concordant behaviours as well as 'abnormal' non-evolutionary-concordant behaviours. Despite significant achievements over the previous century, it is now evident that modern medicine on its own cannot solve all health problems: its resources are over-stretched and its limitations increasingly recognised. Significant progress is no longer solely a matter of eradicating particular diseases, but requires an improved understanding of the political, economic, cultural, social and genetic determinants of health. Improving a nation's health must now rely not just on reactive medical advances but also on positive proactive cultural change that translates directly into improved urban wellbeing.

In the past, public health officials and town planners worked together in the drive to eradicate such urban evils as cholera and typhoid ${ }^{27}$. One of the prime goals for the new generation of cities must be an equally concerted drive to improve urban wellbeing, as the expanding Healthy Cities movement shows ${ }^{28}$. The concepts underlying the evolutionary determinants of health programme could have a major role in that work, realigning policy and practice to better fit our biology. It would be far most cost effective to work with our Palaeolithic genome rather than continuing the losing battle to maintain a lifestyle that is fundamentally bad for us. Although town life is, superficially, the very antithesis of the hunter-gatherers' world, this urban paradox can be at least partially resolved. A solution lies in the adoption of proxy behaviours, environments and townscapes that mimic key elements of the nutrition, daily activity, social interaction and engagement with the natural world that our minds and bodies demand (consciously, unconsciously or subconsciously). Positive attributes of this shared evolutionary past need to be developed, while negative elements must be constrained.

The practices that such applications suggest have been brought together to form a coherent protocol applied to 21st century townscapes and urban lifestyles. This approach is the Eden Protocol, a short-hand term for the evolutionary determinants of health, social behaviour and urban wellbeing. Under its banner, a series of guidelines have been drawn up to progress this concept. Unsurprisingly, many of the elements in the Personal Protocol appear in healthy living guidance issued by public health authorities across the globe, but often in isolation and usually without the Palaeolithic perspective. A more overtly evolutionary focus on such advice,

\footnotetext{
${ }^{24}$ Rydin, Y., Bleahu, A., Davies, M., Groce, N., Scott, I. and Wilkinson, P., 2012 'Shaping cities for health: complexity and the planning of urban environments in the 21st century', The Lancet 379(9831), 2079-2108

${ }^{25}$ WHO 2004 The Global Burden of Disease: 2004 Update. Geneva: World Health Organization

${ }^{26}$ Gardner, B. and Wardle, J., 2012 'The role of health behaviour', in D. French, K. Vedhara, A. Kaptein and J. Weinman (eds), Health Psychology (2nd edn). Oxford: Blackwell, pp. 13-32

${ }^{27}$ Herbert, M., 1999 'A city in good shape: town planning and public health', Town Planning Review 70(4), 433-453

${ }^{28}$ Rydin, Y., Bleahu, A., Davies, M., Groce, N., Scott, I. and Wilkinson, P., 2012 'Shaping cities for health: complexity and the planning of urban environments in the 21st century', The Lancet 379(9831), 2079-2108
} 
however, is apparent in lifestyle literature from, for example, the Primal movement in California ${ }^{29}$ to academics in the United States ${ }^{30}$ and in Europe ${ }^{31}$.

\section{Evolutionary concordance}

The Eden Protocol ${ }^{32}$ incorporates suggestions on how modern-day urban living can better fit our biology - our uncivilised genes - and thus significantly improve our wellbeing. There is clearly some overlap between these levels: some individuals might be tempted to go it alone, perhaps to take up cycling as their daily commute (Personal Protocol), but support in the workplace would obviously be beneficial: a covered, secure cycle rack, for example (Employer's Protocol). Then again, the more your city had designated cycle tracks stripped of heavy lorries turning left, the more likely you would be to cycle (Urban Design Protocol), unless of course, the urban air is still thick with diesel particulates (Political Protocol). But what comes first: the chicken or the egg the bike, the designated cycle track, or the Ultra Low Emissions Zone?

\section{Level 1 Personal protocol}

This is a summary of the key themes in a health behaviour package that most individuals can have, or can develop, control over. Most are based on aspects of a hunter-gatherer lifestyle reworked for a 21st century context. It is not seen as an evolutionary regression but as a return to a normality in keeping with our biology. The first half of the protocol looks at 'good health', which equates with a lifestyle in which our physiology functions normally (ie in an evolutionary-concordant fashion). Should we repeatedly or continuously indulge in abnormal (ie non-evolutionary-concordant behaviours), then 'poor health' will ultimately ensue.

- $\quad$ Eat healthily (eg fresh fruit, vegetables, nuts, fish and lean meat)

- Keep active: walk or cycle all or part of the way to school/work/college whenever possible; use stairs, rather than the lifts, whenever possible.

- Limit smoking and alcohol consumption.

- Spend as much time outdoors as weather permits, preferably in greenspace.

The second half of the list looks at features that relate to our psychological state, underlining the importance of for example, social inclusion.

- $\quad$ Take active part in life of your family and household, and also your community;

- Take an active interest in your town (your territory), its past, its present and its future.

- Challenge yourself physically and mentally: take risks and be prepared to push yourself - essential attributes in the uncertain world of the hunter-gatherer.

Level 2 Institutional Protocols

Working from the themes described in the Personal Protocol, the next three sections look at the responsibilities of institutions such as our employers, our schools and our universities, and suggest some ways in which these bodies might encourage and facilitate positive cultural changes for their staff and students, thereby improving both the health and the productivity of their charges.

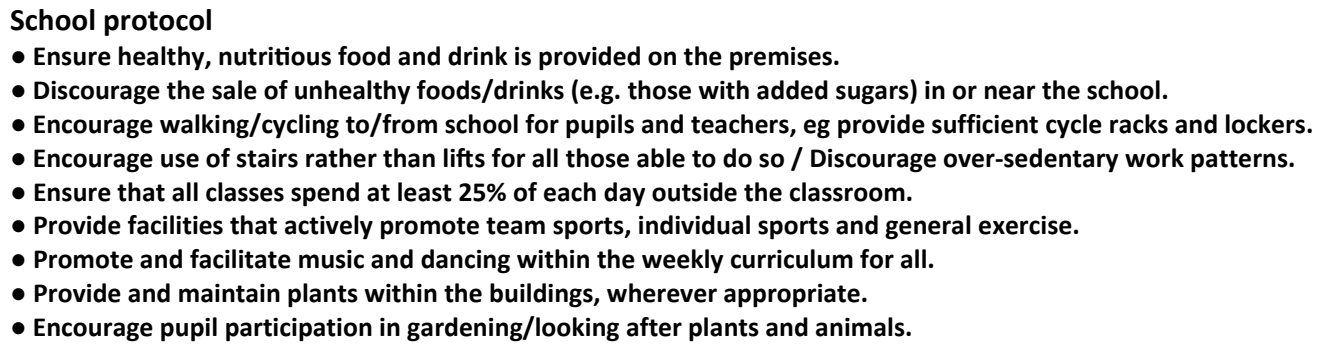

\footnotetext{
${ }^{29}$ Sissons, M., 2009 The Primal Blueprint: Reprogramme Your Genes for Effortless Weight Loss, Vibrant Health and Boundless Energy. Malibu, CA: Primal Nutrition

${ }^{30}$ Cordain, L., Eaton, S. B., Sebastian, A., Mann, N., Lindeberg, S., Watkins, B., O'Keefe, J. and Brand Miller, J., 2005 'Origins and evolution of the Western diet: health implications for the 21st century', American J of Clinical Nutrition 81, 341-354; Konner, M. and Eaton, S. B., 2010 'Palaeolithic nutrition: 25 years later', Nutrition in Clinical Practice 25, 594-602

31 see eg Heylighen, F., 2010 'Evolutionary well-being: the Paleolithic model', ECCO Available at: http://ecco.vub.ac.be/?q=node/127
}

32 Milne, G., 2017 Uncivilised Genes: human evolution and the urban paradox, 208-215 
- Ensure all pupils are challenged academically and physically

University protocol

- Ensure healthy, nutritious food and drink is provided in refectory/ discourage sale of eg foods/drinks with added sugars.

- Encourage active commuting to/from university for staff \& students eg provide sufficient cycle racks, lockers/ showers.

- Encourage the use of stairs rather than lifts for all those able to do so.

- Discourage over-sedentary work patterns.

- Ensure as many courses as possible have field-trip/external element(s).

- Ensure lectures where students are seated do not last beyond one hour without a break.

- Promote and facilitate music, dancing and sport for all, regardless of degree subject.

- Provide attractive public open spaces, suitably planted/ maintained: encourage student participation in campus gardening.

- Provide and maintain plants within the buildings, wherever appropriate: encourage student participation in their tending

\section{Employer protocol}

- Ensure healthy, nutritious food and drink is provided in the canteen.

- Discourage the sale of unhealthy foods/drinks (e.g. those with added sugars).

- Encourage active commuting, eg provide sufficient cycle racks, lockers and showers.

- Encourage use of stairs rather than lifts for all those able to do so.

- Develop new work patterns and office layouts for staff in the most sedentary roles.

- Provide and maintain plants within the buildings, wherever appropriate: encourage staff participation in their tending.

\section{Level 3 Urban Design Protocol}

Consideration of the evolutionary determinants of health provides an integrated rationale underpinning approaches open to local authorities and planning agencies that can facilitate urban wellbeing. Issues include providing a safe, healthy, greened infrastructure, a good standard of building and public realm provision. It is a hybrid of similar thinking developed quite independently by eg the Healthy Cities movement ${ }^{33}$ and New Urbanists ${ }^{34}$, with a radical 21st-century revision of some of Ebenezer's Howard's thinking on garden cities $^{35}$.

- Provide fresh water and sound sanitation systems

- Ensure good air quality (eg limit or ban vehicles that emit diesel particulates)

- Put human locomotion at the heart of transport policy and street design (eg traffic-calming measures, designated cycle tracks, pedestrianisation)

- Develop integrated public transport system that helps limit car use/ Develop pedestrianised public realm/market spaces

- Maintain/ extend urban greenspace/bluespace

- Promote/ develop participatory urban greenspace (allotments, community gardens, city farms).

- Promote active lives with individual and team sport through provision of pitches and facilities.

- Develop programmes of street greening: promote the development of roof gardens etc.

- Limit development for high-rise buildings for residential use (maximum six storeys preferable)

- Encourage street-based neighbourhoods rather than enclosed estates.

- Ensure residential buildings have adequate natural light.

- Ensure all residential units have access to some outside space (garden, patio, terrace or balcony).

Level 4 Political Protocol

This fourth level is one which underwrites the legislation and regulations required to underpin our Protocols and addresses 'downstream' effects suffered by lives blighted by non-evolutionary concordant health behaviours or non-evolutionary town planning, as well as by wider economic, political and social problems. This is essentially reactive, addressing situations that the protocols summarised above aim to alleviate upstream, at least in part. Examples of evolutionary-concordant political interventions would be the so called 'Sugar Taxes', food labelling; sundry measures to constrain smoking or excessive alcohol consumption, the imposition of Ultra Low Emissions Zones aimed at cleaning up urban air in London, to modifying the National Curriculum to ensure school children have adequate knowledge and opportunity to lead 'healthy' lives. An even broader range of challenges to be addressed politically is discussed by Professor Sir Michael Marmot in his 2008 report on the Social Determinants of Health compiled for the World Health Organisation ${ }^{36}$.

\footnotetext{
${ }^{33}$ Tsouros, A., 1990 Healthy Cities Project: A Project Becomes a Movement. Copenhagen: FADL: WHO Healthy Cities Project Office; Hancock, T., 1993 'The evolution, impact and significance of the health cities/health communities movement', Journal of Public Health Policy 14, 5-18.

${ }^{34}$ Congress for New Urbanism (nd) The Charter of the New Urbanism. Available at: https://www.cnu.org/who-weare/charter-new-urbanism.

${ }^{35}$ Howard, E., (1902 [1898]) Garden Cities of Tomorrow (2nd edn). London

${ }^{36}$ WHO 2008 Closing the gap in a generation: Health equity through action on the social determinants of health, Commission on the Social Determinants of Health
} 


\section{Conclusion}

Over many millennia, the human race has adapted to living in forests, valleys, deserts, jungles, mountains and open plains. But can it take the next step in its evolutionary progress, and adapt more successfully to an extensive urbanised environment of its own making? Tomorrow's cities and urban societies would be 'healthier' if configured or reconfigured on evolutionary-concordant principles, working with our palaeolithic genome rather than against it. This would require co-ordinated approaches combining cultural change with changes in urban design, town planning and associated legislation to develop an evolutionary-concordant urban future. Towns may not be our natural habitat, but we can make them our optimal one. 